Research Article

\title{
Application of Adaptive Local Iterative Filtering and Permutation Entropy in Gear Fault Recognition
}

\author{
Wenbin Zhang $\mathbb{D}^{1},{ }^{1}$ Yun Wang, ${ }^{1}$ Yushuo Tan, ${ }^{2}$ Dewei Guo, ${ }^{1}$ and Yasong $P u^{1}$ \\ ${ }^{1}$ College of Engineering, Honghe University, \\ Key Laboratory of Mechanical Performance Analysis and Optimization of Plateau in Yunnan Province, Mengzi, China \\ ${ }^{2}$ Shijiazhuang Post and Telecommunications Technical College, Shijiazhuang, China
}

Correspondence should be addressed to Wenbin Zhang; 190322507@qq.com

Received 4 June 2021; Revised 16 September 2021; Accepted 17 September 2021; Published 11 October 2021

Academic Editor: Yuxing Li

Copyright ( $\odot 2021$ Wenbin Zhang et al. This is an open access article distributed under the Creative Commons Attribution License, which permits unrestricted use, distribution, and reproduction in any medium, provided the original work is properly cited.

In this paper, a fault identification method combining adaptive local iterative filtering and permutation entropy is proposed. The adaptive local iterative filtering can decompose the nonstationary signal into a finite number of stationary intrinsic mode functions. And the experiment gear fault data are decomposed into several intrinsic mode functions by this method. Then, using the permutation entropy to calculate each intrinsic mode function, it is found that the permutation entropy of the first several intrinsic mode functions can represent the characteristics of different fault types, and the permutation entropy of the intrinsic mode function corresponding to the rotating frequency signal of the gear system could be the boundary. Finally, the fault type of gear is identified by calculating the gray correlation degree of permutation entropy of essential mode function of vibration signal decomposition under different working conditions. The example analysis results show that the proposed method can be effectively applied to the fault diagnosis of the gear system.

\section{Introduction}

Gearbox is the main part of mechanical equipment for motion and power transmission. In gear transmission, machine failure caused by gear failure will cause equipment shutdown or even damage. Therefore, how to extract the gear fault feature parameters effectively has been a research hot spot of many scholars [1, 2]. Since Huang et al. [3] proposed empirical mode decomposition, the empirical mode decomposition method has attracted the attention of many scholars. It extends from the traditional orthogonal basis function expansion to the data-driven signal representation with adaptive ability for any complex signal. However, the empirical mode decomposition (EMD) method has some problems, such as mode confusion caused by singular points in the signal and instability under noise interference. Inspired by the idea of empirical mode decomposition, some scholars have proposed a new adaptive mode decomposition method, among which adaptive local iterative filtering [4] is a representative one. Based on the idea of the empirical mode decomposition (EMD) method, the adaptive local iterative filtering method adopts the Fokker-Planck equation to design the filter, which can effectively avoid false components in the decomposition process and is more suitable for the analysis of nonlinear and nonstationary signals. Deyou et al. [5] applied the adaptive local iterative filtering method to feature extraction of nonstationary signals in the power system and achieved good results. Baojia et al. [6] applied this method to fault feature extraction of rolling bearing. However, in the fault feature extraction of the gear system, the research of the adaptive local iterative filtering method is still less. The results show that the gear fault signal has typical nonstationary and nonlinear characteristics. Therefore, how to effectively extract the fault features reflecting different working conditions of gears is extremely important [7].

Entropy is a powerful tool for the analysis of time series as it allows describing the probability distributions of the possible state of a system and therefore the information encoded in it. Entropy is a basic quantity with multiple field- 
specific interpretations; for instance, it has been associated with disorder, state-space volume, or lack of information. When dealing with information content, the Shannon entropy is often considered as the foundational and most natural one. Nevertheless, important information may be codified also in the temporal dynamics, an aspect which is not usually taken into account. The idea of calculating entropy based on permutation patterns (that is, permutations defined by the order relations among values of a time series) has received a lot of attention in the last years. Permutation entropy is an information entropy for studying the complexity of nonlinear time series. Compared with the commonly used nonlinear dynamic methods, such as Lyapunov index, fractal dimension, and sample entropy, permutation entropy has good antinoise performance. Moreover, the time series required is short, the calculation is simple, and the operation speed is fast [8-11].

In this paper, adaptive local iterative filter decomposition, combined with permutation entropy and gray correlation degree, is used to classify and identify different fault types of the gear system. Firstly, the normal, mild wear, moderate wear, and broken teeth are simulated on the gearbox simulation experimental platform. Then, adaptive local iterative filtering is used to decompose the sampled signal adaptively. Then, the permutation entropy of each intrinsic mode function component is calculated. Finally, the fault recognition and classification are carried out by calculating the gray correlation degree between the samples to be identified and the standard failure modes. The results of case analysis show that the method can be used to identify different fault types of the gear system effectively.

\section{Principle of Adaptive Local Iterative Filtering Technique}

In empirical mode decomposition, the instantaneous mean is defined as the mean function of the upper and lower envelopes. Because the cubic spline which is sensitive to singular points is used to connect the local maximum and local minimum to fit the upper and lower envelopes, the instantaneous mean is unstable under disturbance. In order to overcome this defect, Lin et al. [12] proposed an iterative filtering algorithm in 2009. The algorithm follows the same framework as empirical mode decomposition (EMD), but the instantaneous mean is obtained by low-pass filtering. In order to ensure the stability and convergence under disturbance, the uniform double length average filter is used in iterative filtering. However, the filter is not smooth enough and may cause false fluctuations in the intrinsic mode function. In order to overcome this defect, Cicone et al. [4] proposed an adaptive local iterative filtering algorithm in 2016. This method extends the iterative filtering algorithm by using the nonuniform filter. The filter is designed as the Fokker-Planck equation, as shown in equation (1), so that the filter has compact support in the time domain and the length is flexible, which can avoid false fluctuations in the iterative filtering process.

$$
\frac{\partial}{\partial t} g(x, t)=-\alpha \frac{\partial}{\partial x}[p(x, t) g(x, t)]+\beta \frac{\partial^{2}}{\partial x^{2}}\left[q^{2}(x, t) g(x, t)\right] .
$$

In the formula [4], the value range of $\alpha$ and $\beta$ is between $(0,1)$.

The implementation process of the adaptive local iterative filtering algorithm is as follows [13]:

(1) Initialization: let the iteration number $i=1$ and the residual signal is $r_{0}(t)=x(t)$.

(2) The $i$ th intrinsic mode function is extracted.

(2.1) Let the screening number $j=0$ and the prototype intrinsic mode function is $h_{i j}(t)=r_{i-1}$ $(t)$.

(2.2) The adaptive local Fokker-Planck filter $g_{i j}(t, \tau)$ is designed to determine the corresponding time-varying filter length $l_{i j}(t)$.

(2.3) Calculate the instantaneous mean value.

$$
m_{i j}(t)=\int_{-l_{i j}(t)}^{l_{i j}(t)} h_{i j}(t+\tau) g_{i j}(t, \tau) \mathrm{d} \tau .
$$

(2.4) Update the prototype intrinsic mode function.

$$
h_{i j}(t)=h_{i j}(t)-m_{i j}(t)
$$

(2.5) If the prototype intrinsic mode function $h_{i j}(t)$ satisfies the condition requirements of the intrinsic mode function, then let the $i^{\text {th }}$ IMF intrinsic mode function be

$$
c_{i}(t)=h_{i j}(t) .
$$

Otherwise, let the screening times be $j=j+1$ and return to step (2.2).

(3) Update residual signal.

$$
r_{i}(t)=r_{i-1}(t)-c_{i}(t) .
$$

(4) If the residual signal $r_{i}(t)$ satisfies the termination criterion of the algorithm, that is, only one extreme point becomes the trend term, the adaptive local iterative filtering decomposition is terminated. Otherwise, let the iteration number $i=i+1$ and return to step (2).

\section{Definition of Permutation Entropy}

Let the sampling sequence be $[x(n)]=x(1), x(2), \ldots, x(N)$. The sequence permutation entropy $[8-11]$ is calculated as follows:

(1) By reconstructing the phase space of the sampling sequence, the following results can be obtained:

$$
X(i)=[x(i), x(i+\tau), \ldots, x(i+(m-1) \tau)] .
$$

In formula, $m$ and $\tau$ are embedding dimension and delay time, respectively.

(2) $M$ data of $X(i)$ are arranged in ascending order: 
$x\left(i+\left(j_{1}-1\right) \tau\right) \leq x\left(i+\left(j_{2}-1\right) \tau\right) \leq \ldots \leq x\left(i+\left(j_{m}-1\right) \tau\right)$.

In the formula, $j_{1}, j_{2}, \ldots, j_{m}$ is the location index of the elements after $X(i)$ reordering.

(3) If two elements of equal size exist in $X(i)$, that is,

$$
x\left(i+\left(j_{1}-1\right) \tau\right)=x\left(i+\left(j_{2}-1\right) \tau\right),
$$

they are sorted by the size of the location index value. If $j_{1}<j_{2}$, then

$$
x\left(i+\left(j_{1}-1\right) \tau\right)<x\left(i+\left(j_{2}-1\right) \tau\right) .
$$

(4) For any reconstructed signal $X(i)$, a group of sequences in ascending order can be obtained:

$$
s(g)=\left(j_{1}, j_{2}, \ldots, j_{m}\right) .
$$

In the formula, $g=1,2, \ldots, k$, and $k \leq m$ ! Therefore, $m$-dimensional phase space reconstruction of different sequences total is $m$ ! Sequence $s(g)$ is one of the permutations.

(5) Calculate the probability of occurrence of each sequence, then

$$
P_{i}=\frac{l}{k} \text {. }
$$

In the formula, $l$ is the frequency of $s(g)$, and $l$ does not change with $g$.

(6) In the form of Shannon entropy, the permutation entropy can be defined as

$$
H_{p}(m, \tau)-\sum_{j=1}^{k} P_{i} \operatorname{In} P_{i} .
$$

(7) After normalization of $H_{P}(m, \tau)$, the following results can be obtained [11]:

$$
H_{p}=\frac{H_{p}(m, \tau)}{\operatorname{In}(m !)} .
$$

According to the definition of permutation entropy, the value range of $H_{P}$ is $[0,1]$. The random degree of signal can be expressed by the size of permutation entropy. The smaller the permutation entropy is, the more regular the signal is; otherwise, the more complex it is. The permutation entropy of the sampled signal can be obtained, and the local subtle change of the signal can be detected by the change of its value.

\section{Theory of Gear Fault Recognition Based on Adaptive Local Iterative Filtering and Permutation Entropy}

The principle of gear fault identification is shown in Figure 1. In the gear test system, the fault states of normal gear, slight wear of tooth surface, moderate wear of tooth surface, and broken tooth are simulated. When the gray correlation degree is used for fault type identification, the standard fault mode with the largest gray correlation degree with the sample to be identified is considered as the fault type of the sample to be identified [14]. The detailed process of gray correlation degree identification can be seen in the author's previous research results [15].

\section{Case Analysis}

In order to verify the recognition effect of the proposed method for gear fault, we use the experiment platform to acquire the gear fault data. The experimental platform is shown in Figure 2. The gear rotation frequency is $f_{r}=23.6 \mathrm{~Hz}$, the meshing frequency is $f_{z}=686 \mathrm{~Hz}$, and the vibration signal sampling frequency is $16384 \mathrm{~Hz}$. Due to the limited experiment condition, we only have four common gear working conditions, such as normal gear, slight wear of tooth surface, moderate wear of tooth surface, and broken tooth which are tested on the gear system test platform. Four kinds of gear working conditions are sampled, respectively, 20 samples are taken for each, and the adaptive local iterative filter is used to decompose the sampling signal adaptively. Now, we select one data of each working condition to decompose by adaptive local iterative filtering, and we can see that the decomposition results obtain 8 IMF components and 1 residual component, as shown in Figures 3-6.

It can be seen from the figures that the nonstationary gear fault signal is decomposed into several stationary IMF components by adaptive local iterative filtering. In IMF8 component, the obvious periodic component can be seen. After calculating the frequency of this component, it can be seen that this component corresponds to the gear frequency signal. Limited to space, Figure 7 shows the result of adaptive decomposition of the medium-worn signal by the EEMD method for comparison. In EEMD decomposition, although noise is added in the decomposition process to reduce the degree of modal aliasing, compared with the adaptive local iterative filter decomposition, the mode aliasing phenomenon is more obvious. Moreover, in the decomposition result of EEMD, the rotational frequency component of gear cannot be seen. It also shows that the adaptive iterative filter can effectively suppress the mode aliasing due to the introduction of the Fokker-Planck equation.

In order to further highlight the adaptive decomposition ability of the adaptive local iterative filter, the results of EMD decomposition of the same signal are shown in Figure 8. Compared with Figures 5, 7, and 8, it can be seen that $12 \mathrm{IMF}$ components and 1 residual component are obtained by EMD decomposition. EEMD decomposition results in 11 IMF components and 1 residual component. However, eight IMF components and one residual component are obtained by adaptive local iterative filter decomposition. From the point of view of the degree of mode aliasing of the decomposed signal, the EEMD method reduces the degree of modal aliasing to a certain extent by adding white noise in 


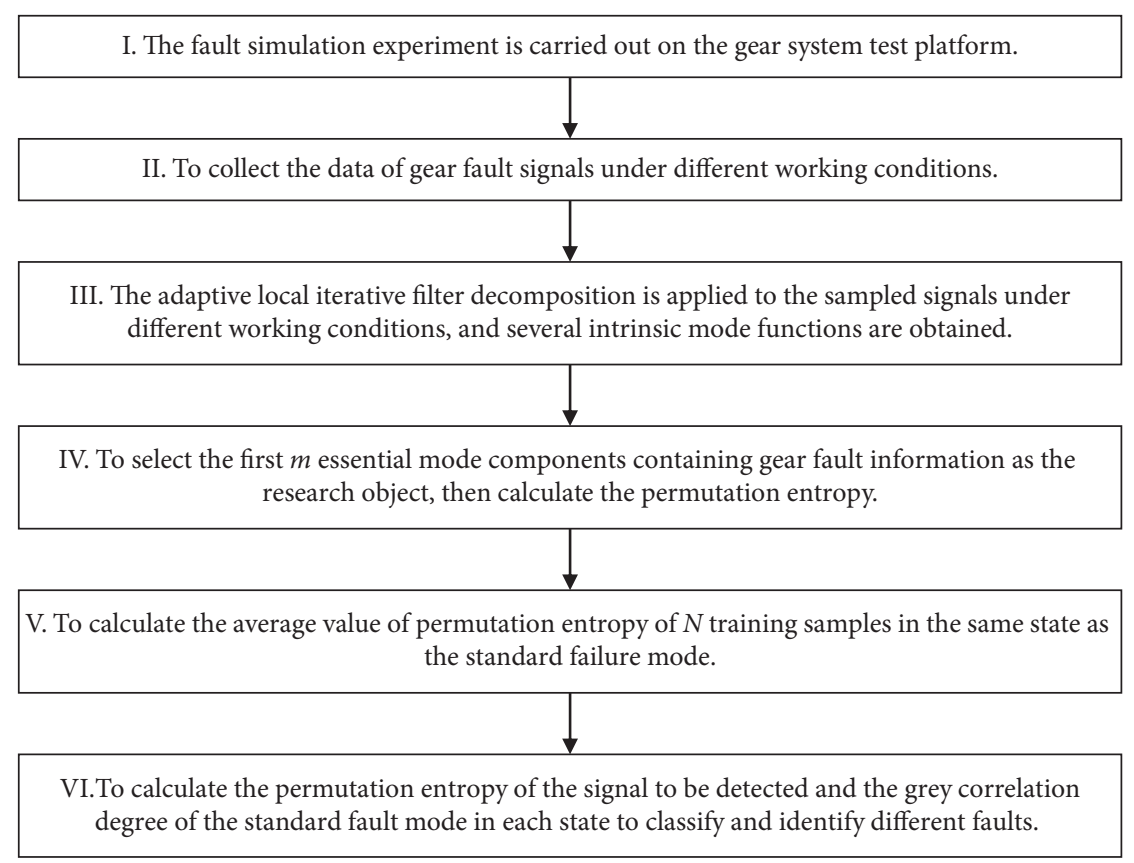

FIGURE 1: Gear fault recognition principle based on adaptive local iterative filter decomposition.

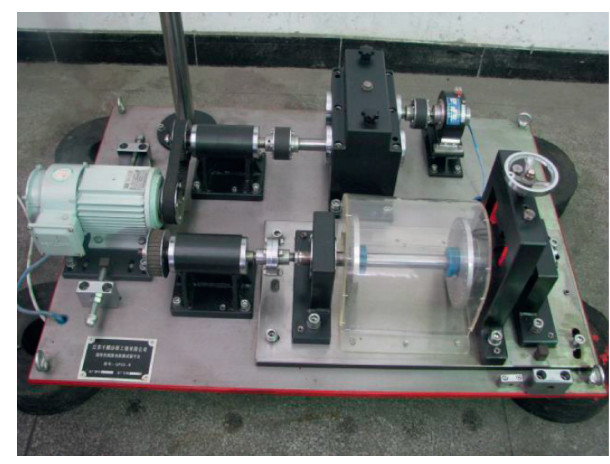

FIGURE 2: Gearbox simulation experiment platform.

the decomposition process on the basis of EMD decomposition. In the signal obtained by adaptive local iterative filtering, the degree of mode aliasing is effectively improved, which is more conducive to the fault feature extraction of the gear system.

Now, 10 samples of each state are randomly selected as training samples. According to the principle steps of fault identification shown in Figure 1, the permutation entropy of 8 intrinsic mode functions obtained by decomposition of each state is calculated, as shown in Figure 9. The value in the figure is the average value of permutation entropy of 10 training samples. It can be seen from the figure that the shape of permutation entropy curve under four conditions is relatively similar. Since the size of permutation entropy reflects the randomness of signals, it can be seen that with the continuous decomposition of signals, the components contained in the components tend to be simple, so the permutation entropy tends to decrease gradually. Since IMF8 corresponds to gear rotation frequency, it can be considered that the first seven IMF contain gear fault information, which shows that the permutation entropy can effectively represent the change of gear fault characteristics. The permutation entropy of the first seven IMF components is taken as the fault feature. Since the permutation entropy curves of the first seven IMF components are similar under the four working conditions, it is very important to select an appropriate identification method to effectively distinguish different fault types in subsequent fault classification and identification.

Due to the space limitation, 5 samples are randomly selected from the remaining 10 samples of each state as the samples to be tested. Table 1 shows the permutation entropy of the samples to be tested.

Finally, the gray correlation degree between the permutation entropy of the sample to be tested and the average value of the permutation entropy of the training sample in each state is calculated by using the gray correlation degree method. According to the value of correlation degree, the fault modes of the gear system are classified and identified. The results are shown in Table 2.

It can be seen from Table 2 that the method of gray correlation degree has achieved ideal results for the fault pattern recognition of the gear system. There is a big difference between the gray correlation degree of corresponding fault types and that of other fault types. In the permutation entropy curve of Figure 6, the shape of permutation entropy curve under four working conditions is similar. Through the method of gray correlation degree, four different fault types can be effectively classified, which shows that the gray correlation degree can accurately classify the problem of small sample fault identification. The correct classification results can also be obtained by identifying the remaining fault samples. 


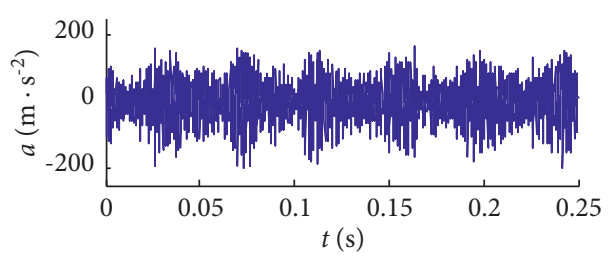

(a)

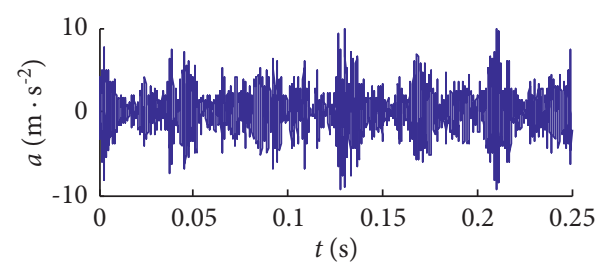

(c)

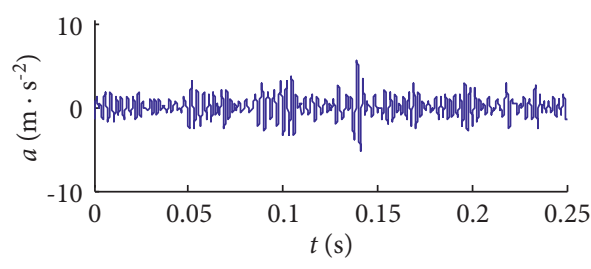

(e)

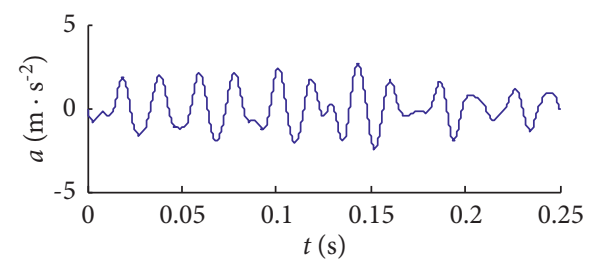

(g)

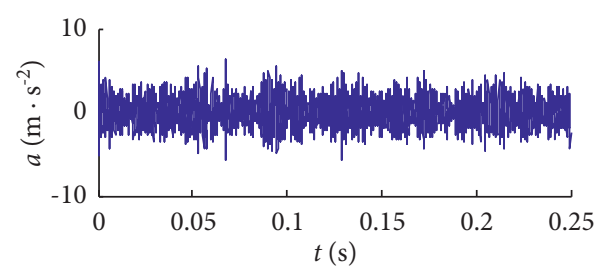

(b)

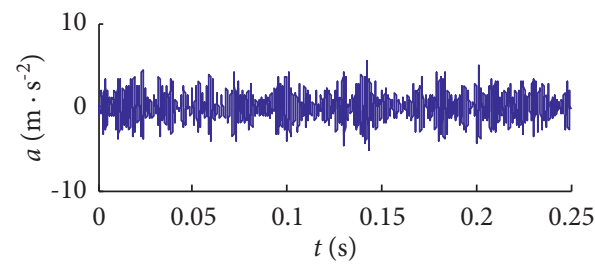

(d)

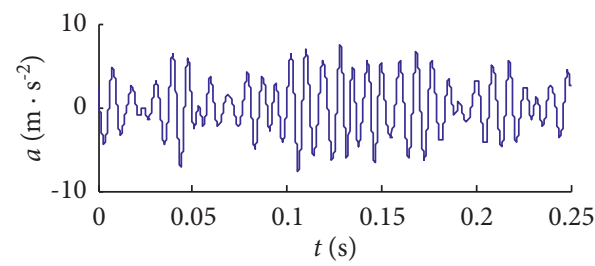

(f)

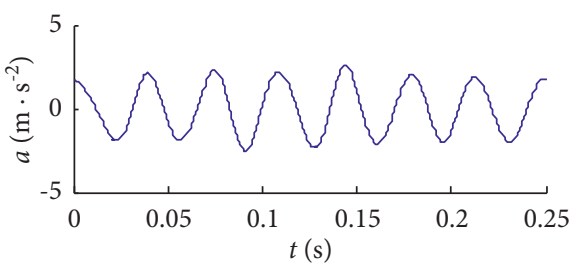

(h)

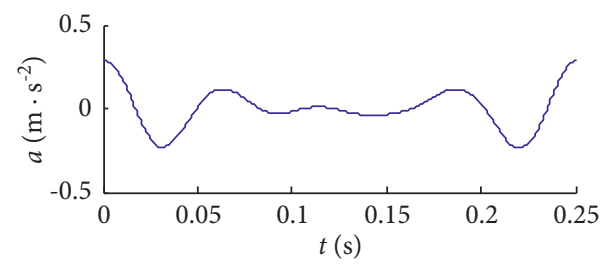

(i)

FIGURE 3: Decomposition results of normal signal by adaptive local iterative filtering: (a) IMF1; (b) IMF2; (c) IMF3; (d) IMF4; (e) IMF5; (f) IMF6; (g) IMF7; (h) IMF8; (i) Ra.

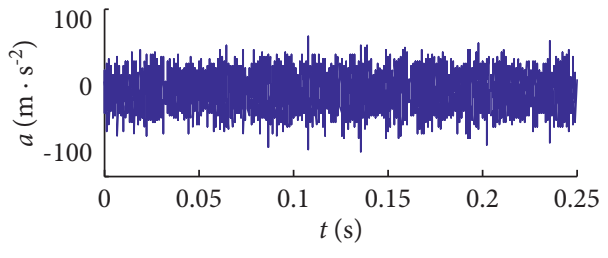

(a)

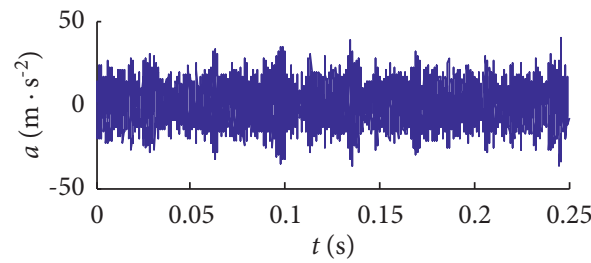

(b)

Figure 4: Continued. 


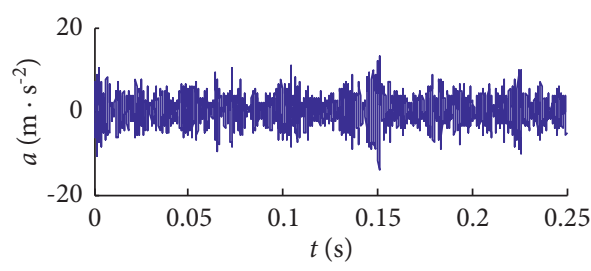

(c)

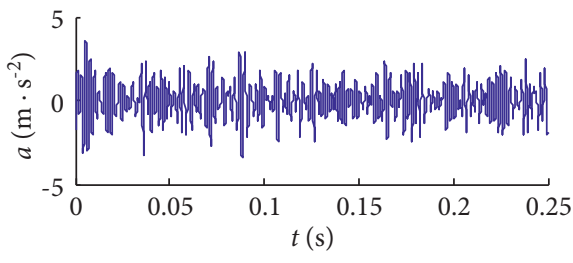

(e)

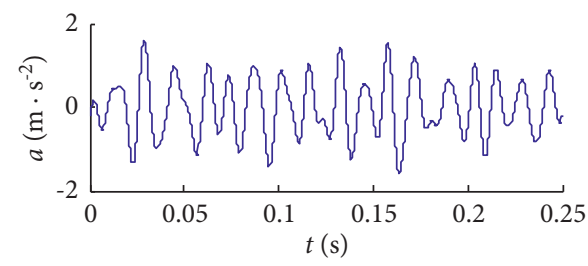

(g)

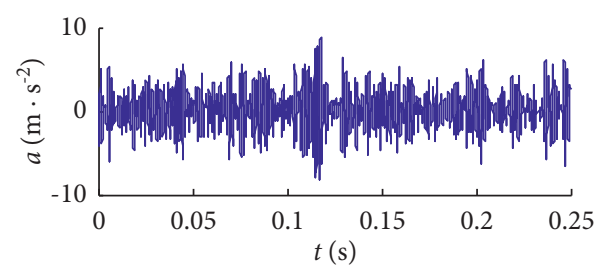

(d)

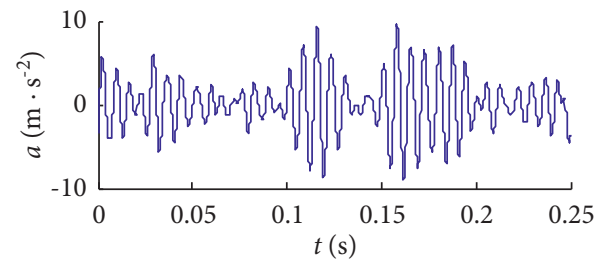

(f)

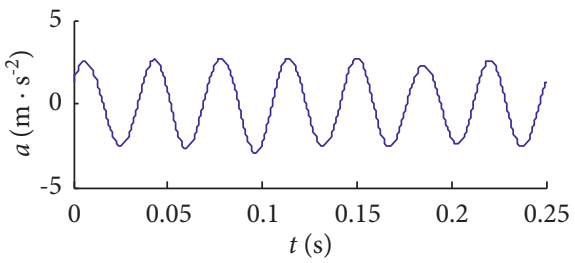

(h)

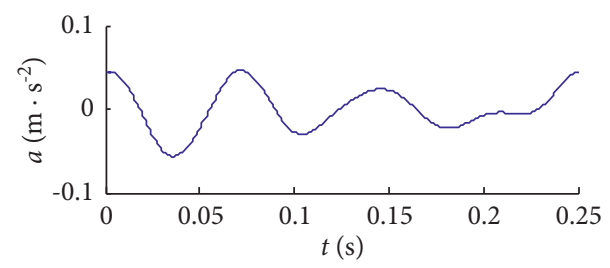

(i)

Figure 4: Decomposition results of slight-worn signal by adaptive local iterative filtering: (a) IMF1; (b) IMF2; (c) IMF3; (d) IMF4; (e) IMF5; (f) IMF6; (g) IMF7; (h) IMF8; (i) Ra.

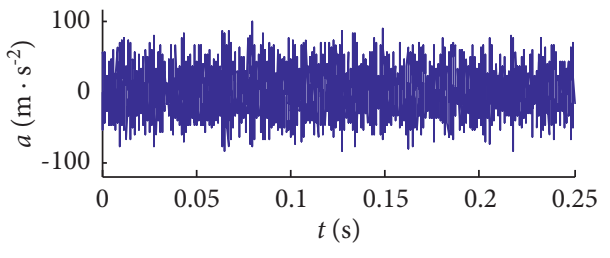

(a)

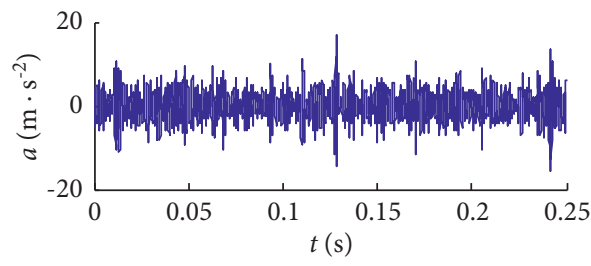

(c)

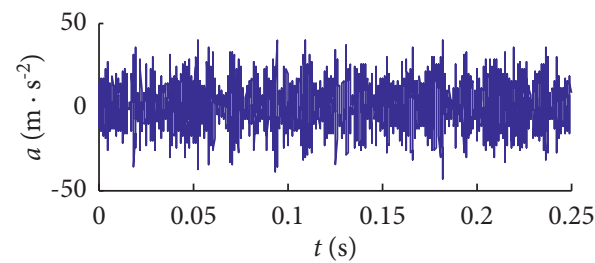

(b)

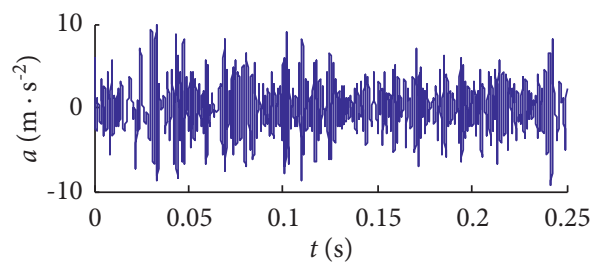

(d)

Figure 5: Continued. 


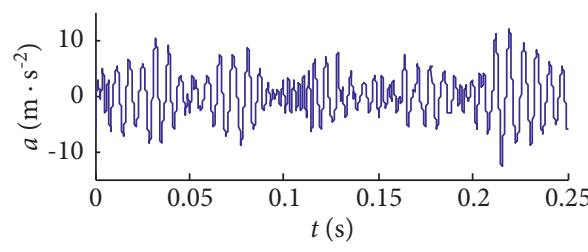

(e)

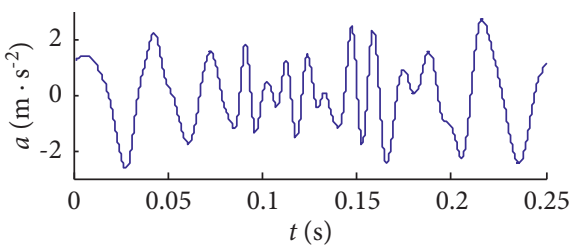

(g)

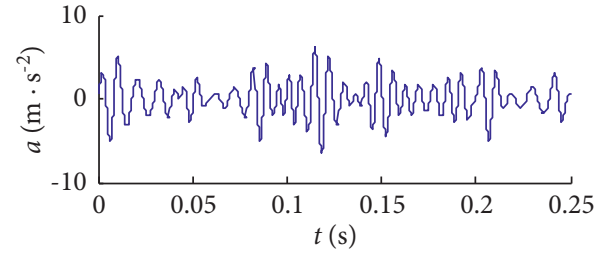

(f)

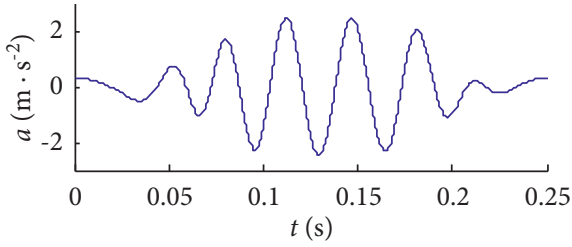

(h)

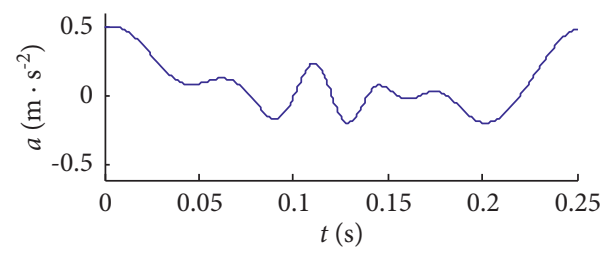

(i)

FIGURE 5: Decomposition results of medium-worn signal by adaptive local iterative filtering: (a) IMF1; (b) IMF2; (c) IMF3; (d) IMF4; (e) IMF5; (f) IMF6; (g) IMF7; (h) IMF8; (i) Ra.

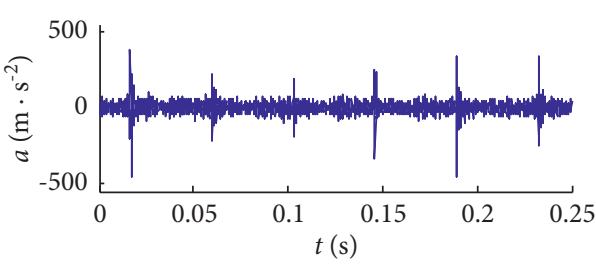

(a)

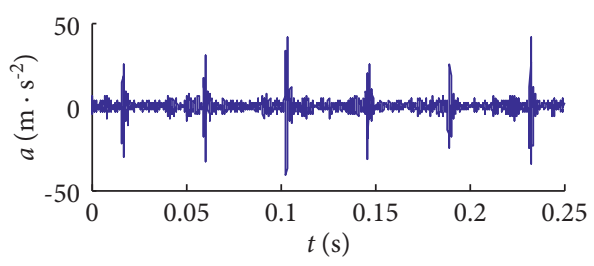

(c)

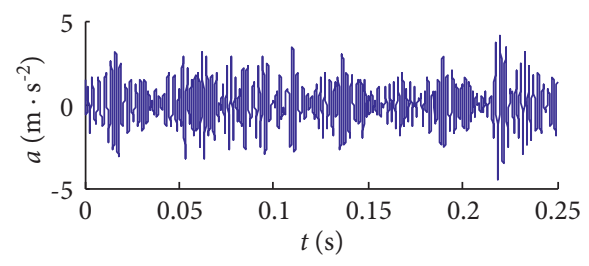

(e)

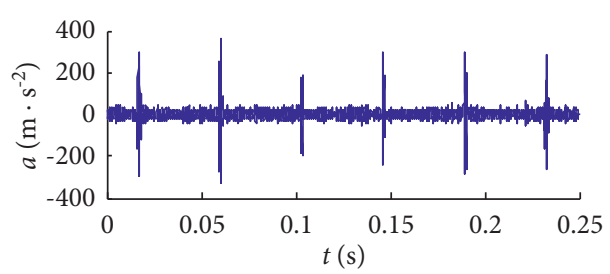

(b)

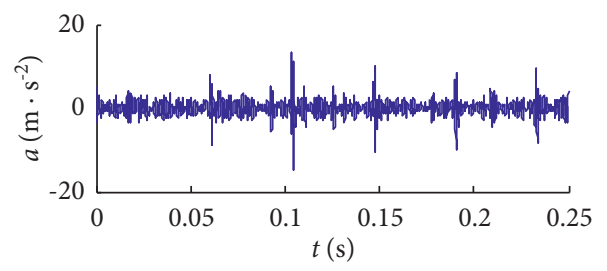

(d)

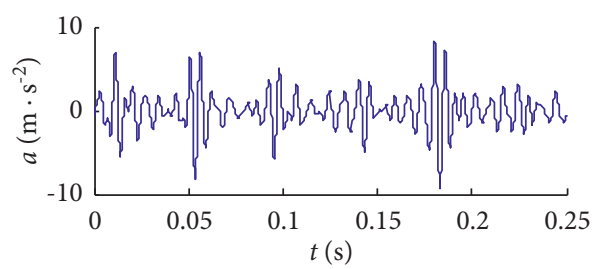

(f)

Figure 6: Continued. 


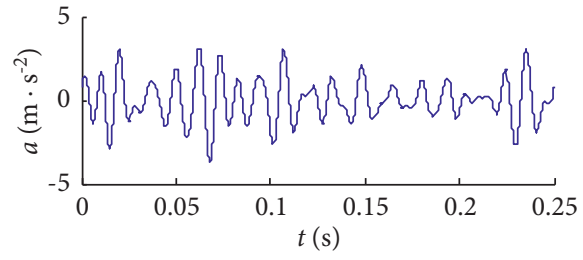

(g)

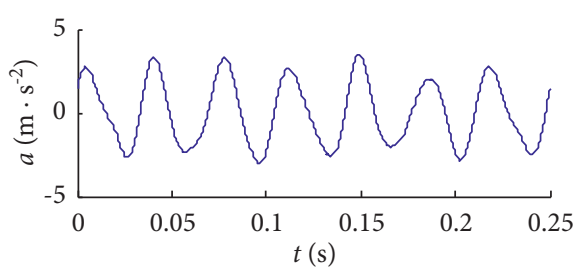

(h)

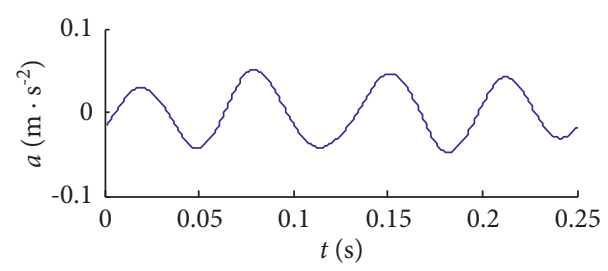

(i)

FIGURE 6: Decomposition results of broken-tooth signal by adaptive local iterative filtering: (a) IMF1; (b) IMF2; (c) IMF3; (d) IMF4; (e) IMF5; (f) IMF6; (g) IMF7; (h) IMF8; (i) Ra.

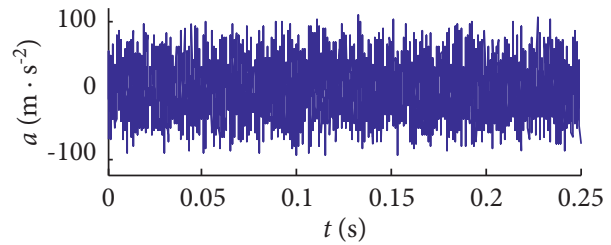

(a)

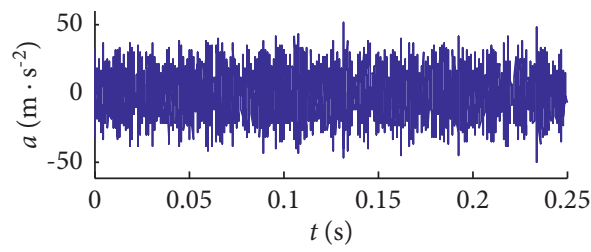

(c)

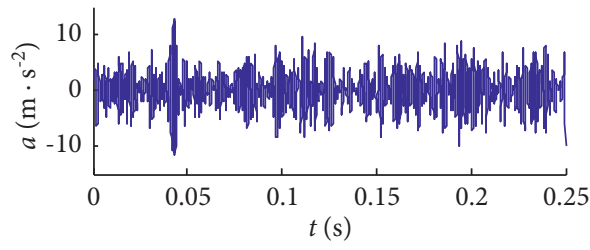

(e)

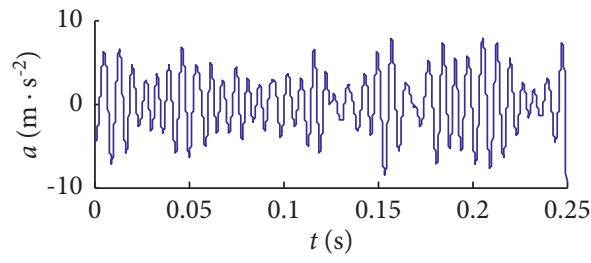

(g)

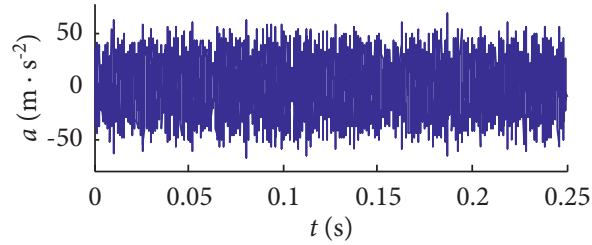

(b)

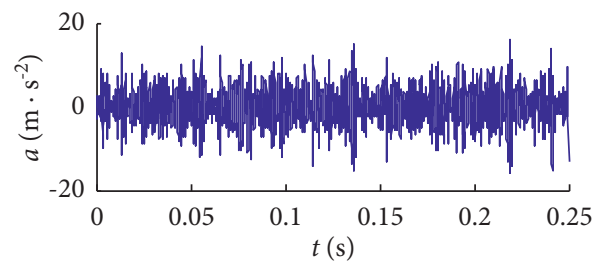

(d)

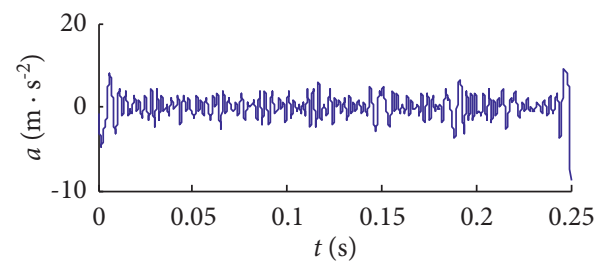

(f)

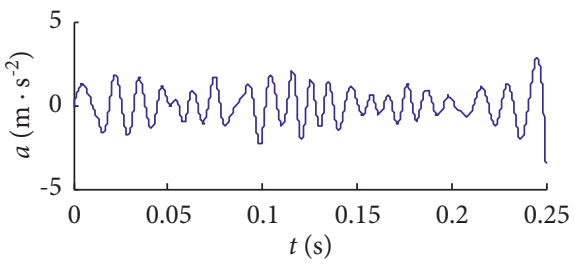

(h)

Figure 7: Continued. 


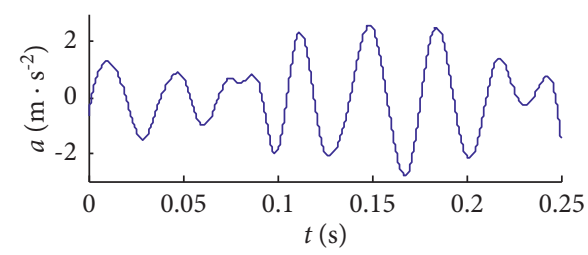

(i)

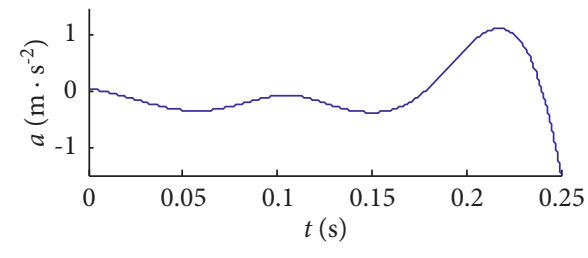

(k)

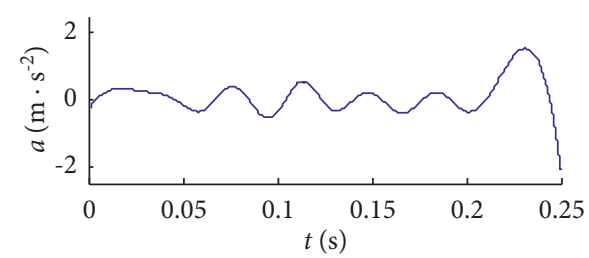

(j)

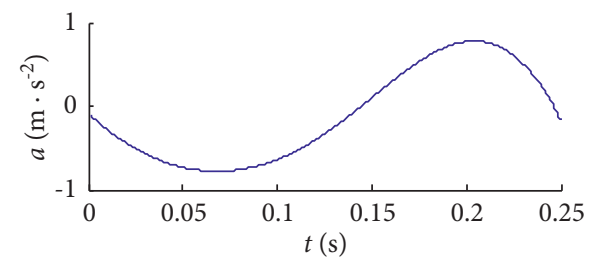

(l)

FIGURE 7: Decomposition results of medium-worn signal by EEMD: (a) IMF1; (b) IMF2; (c) IMF3; (d) IMF4; (e) IMF5; (f) IMF6; (g) IMF7; (h) IMF8; (i) IMF9; (j) IMF10; (k) IMF11; (l) Ra.

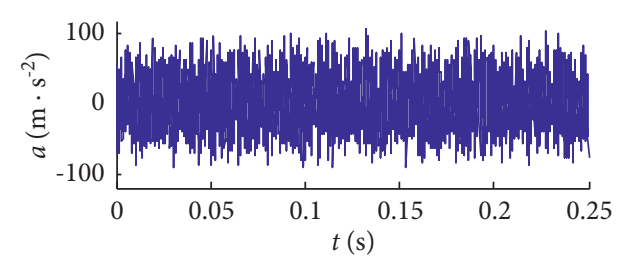

(a)

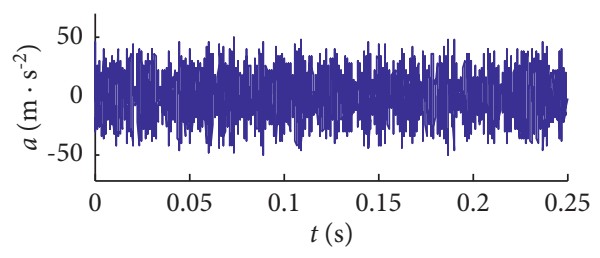

(c)

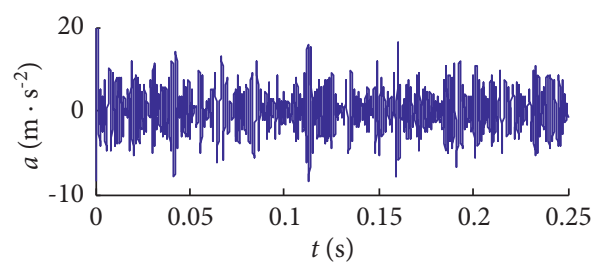

(e)

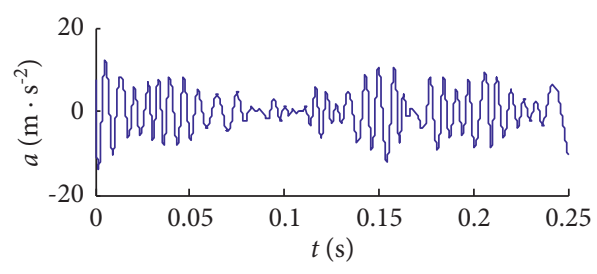

(g)

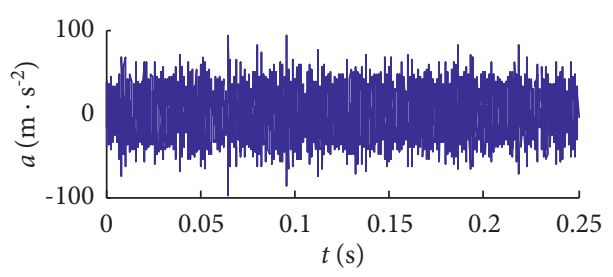

(b)

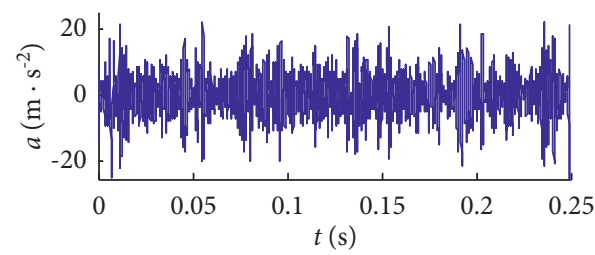

(d)

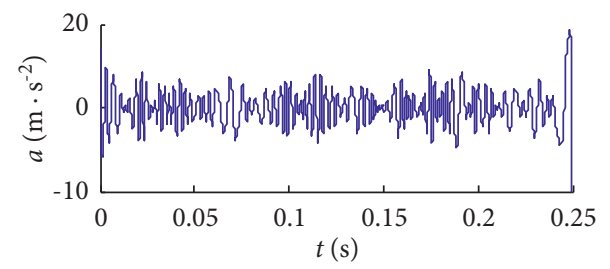

(f)

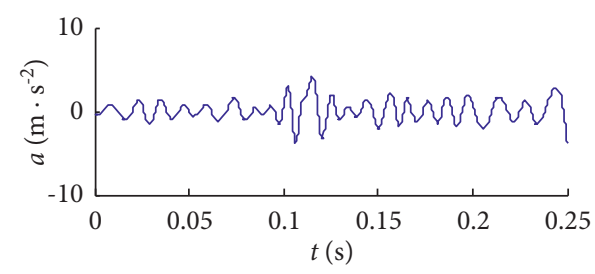

(h)

FIGURE 8: Continued. 


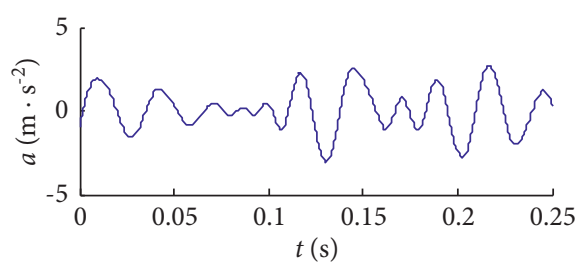

(i)

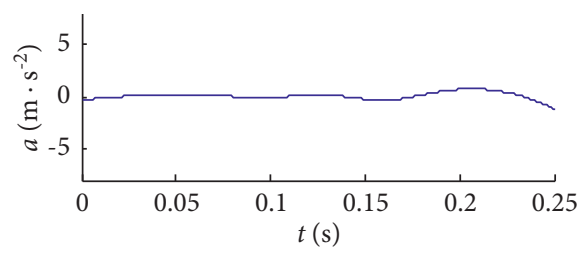

(k)

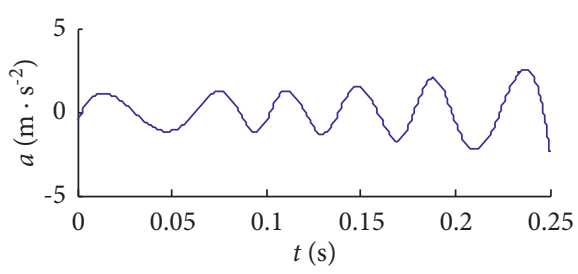

(j)

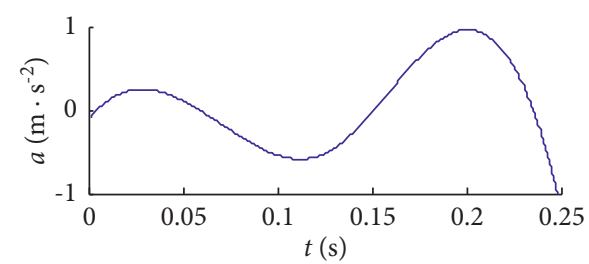

(l)

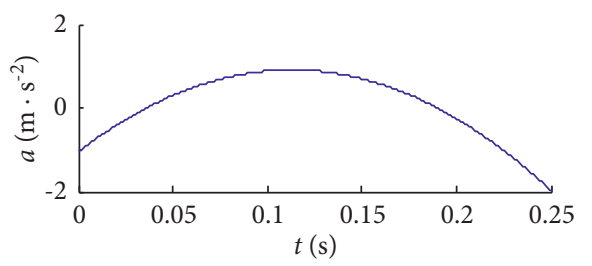

(m)

FIGURE 8: Decomposition results of medium-worn signal by EMD: (a) IMF1; (b) IMF2; (c) IMF3; (d) IMF4; (e) IMF5; (f) IMF6; (g) IMF7; (h) IMF8; (i) IMF9; (j) IMF10; (k) IMF11; (l) IMF12; (m) Ra.

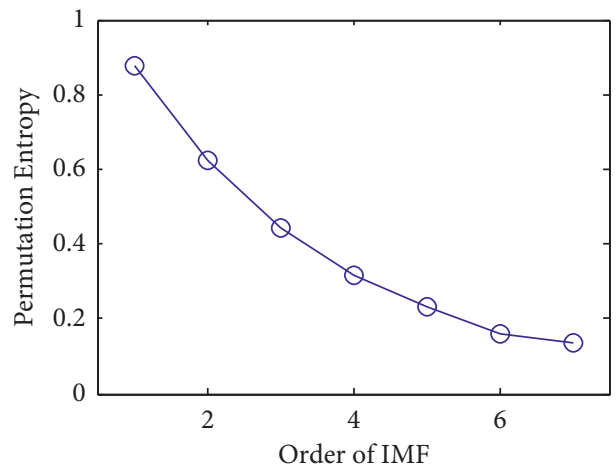

(a)

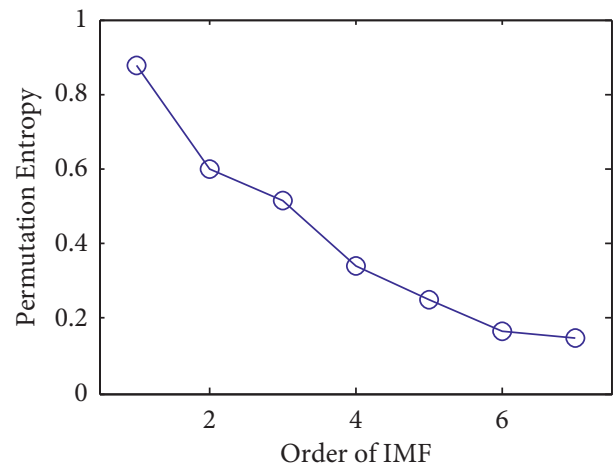

(c)

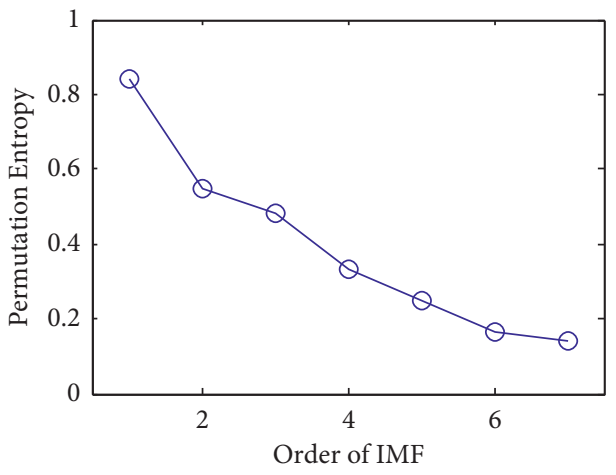

(b)

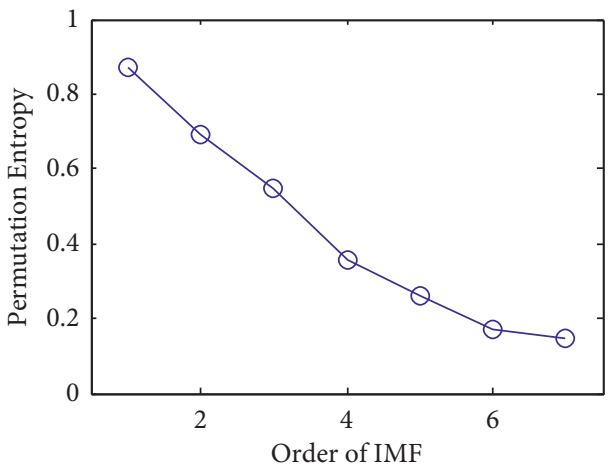

(d)

FiguRE 9: Permutation entropy curve of the gear system under different working conditions: (a) normal gear; (b) slight wear of tooth surface; (c) moderate wear of tooth surface; (d) broken tooth. 
TABle 1: Permutation entropy of the gear system under different working conditions.

\begin{tabular}{|c|c|c|c|c|c|c|c|c|}
\hline Working condition & Sample & $H_{p 1}$ & $H_{p 2}$ & $H_{p 3}$ & $H_{p 4}$ & $H_{p 5}$ & $H_{p 6}$ & $H_{p 7}$ \\
\hline \multirow{5}{*}{ Normal gear } & 1 & 0.8710 & 0.6331 & 0.4395 & 0.3143 & 0.2395 & 0.1663 & 0.1394 \\
\hline & 2 & 0.8727 & 0.6264 & 0.4423 & 0.3177 & 0.2316 & 0.1593 & 0.1344 \\
\hline & 3 & 0.8709 & 0.6321 & 0.4481 & 0.3197 & 0.2384 & 0.1596 & 0.1364 \\
\hline & 4 & 0.8780 & 0.6259 & 0.4400 & 0.3240 & 0.2362 & 0.1593 & 0.1363 \\
\hline & 5 & 0.8711 & 0.6224 & 0.4375 & 0.3090 & 0.2342 & 0.1592 & 0.1379 \\
\hline \multirow{5}{*}{ Slight wear of tooth surface } & 1 & 0.8379 & 0.5444 & 0.4829 & 0.3301 & 0.2479 & 0.1650 & 0.1392 \\
\hline & 2 & 0.8371 & 0.5577 & 0.4796 & 0.3295 & 0.2550 & 0.1616 & 0.1373 \\
\hline & 3 & 0.8328 & 0.5488 & 0.4765 & 0.3258 & 0.2456 & 0.1613 & 0.1377 \\
\hline & 4 & 0.8408 & 0.5419 & 0.4766 & 0.3305 & 0.2552 & 0.1687 & 0.1448 \\
\hline & 5 & 0.8390 & 0.5453 & 0.4842 & 0.3243 & 0.2482 & 0.1665 & 0.1413 \\
\hline \multirow{5}{*}{ Moderate wear of tooth surface } & 1 & 0.8748 & 0.5980 & 0.5124 & 0.3345 & 0.2511 & 0.1700 & 0.1478 \\
\hline & 2 & 0.8795 & 0.5994 & 0.5079 & 0.3340 & 0.2478 & 0.1697 & 0.1478 \\
\hline & 3 & 0.8752 & 0.6008 & 0.5151 & 0.3461 & 0.2479 & 0.1664 & 0.1473 \\
\hline & 4 & 0.8826 & 0.6015 & 0.5177 & 0.3284 & 0.2486 & 0.1691 & 0.1474 \\
\hline & 5 & 0.8786 & 0.5948 & 0.5121 & 0.3380 & 0.2531 & 0.1681 & 0.1441 \\
\hline \multirow{5}{*}{ Broken tooth } & 1 & 0.8633 & 0.6903 & 0.5470 & 0.3574 & 0.2586 & 0.1754 & 0.1520 \\
\hline & 2 & 0.8693 & 0.6883 & 0.5446 & 0.3543 & 0.2581 & 0.1668 & 0.1459 \\
\hline & 3 & 0.8705 & 0.6939 & 0.5497 & 0.3486 & 0.2576 & 0.1681 & 0.1461 \\
\hline & 4 & 0.8734 & 0.6822 & 0.5510 & 0.3561 & 0.2548 & 0.1682 & 0.1449 \\
\hline & 5 & 0.8651 & 0.6909 & 0.5452 & 0.3480 & 0.2597 & 0.1683 & 0.1442 \\
\hline
\end{tabular}

TABLe 2: Gray relation degree between tested samples and standard fault patterns.

\begin{tabular}{lccccc}
\hline Sample & Normal gear & Slight wear of tooth surface & Moderate wear of tooth surface & Broken tooth & Result \\
\hline 1 & 0.9047 & 0.7927 & 0.7750 & 0.6951 & Normal gear \\
2 & 0.9431 & 0.7684 & 0.7340 & 0.6716 & Normal gear \\
3 & 0.9339 & 0.7670 & 0.7457 & 0.6694 & Normal gear \\
4 & 0.9419 & 0.7727 & 0.7575 & 0.6635 & Normal gear \\
5 & 0.9420 & 0.7578 & 0.7408 & 0.6617 & Normal gear \\
6 & 0.7802 & 0.9670 & 0.8051 & 0.7081 & Slight wear of tooth surface \\
7 & 0.7937 & 0.9022 & 0.7762 & 0.6853 & Slight wear of tooth surface \\
8 & 0.8176 & 0.9244 & 0.7708 & 0.6818 & Slight wear of tooth surface \\
9 & 0.7438 & 0.9102 & 0.8227 & 0.7550 & Slight wear of tooth surface \\
10 & 0.7523 & 0.9309 & 0.7914 & 0.6914 & Slight wear of tooth surface \\
11 & 0.6626 & 0.7026 & 0.9144 & 0.7109 & Moderate wear of tooth surface \\
12 & 0.7060 & 0.7819 & 0.9149 & 0.7252 & Moderate wear of tooth surface \\
13 & 0.6856 & 0.7524 & 0.9017 & 0.7096 & Moderate wear of tooth surface \\
14 & 0.6893 & 0.7353 & 0.8994 & 0.7140 & Moderate wear of tooth surface \\
15 & 0.6952 & 0.7404 & 0.9298 & 0.9286 & Broken tooth surface \\
16 & 0.6694 & 0.7064 & 0.7616 & 0.9384 & Broken tooth \\
17 & 0.7115 & 0.7489 & 0.8161 & 0.9360 & Broken tooth \\
18 & 0.7194 & 0.7572 & 0.8340 & 0.9161 & Broken tooth \\
19 & 0.7180 & 0.7534 & 0.8204 & 0.9256 & Broken tooth \\
20 & 0.7006 & 0.7465 & 0.8002 & & \\
\hline
\end{tabular}

\section{Conculsion}

In this paper, the adaptive local iterative filtering method is used to decompose the gear fault data. The method can decompose the nonstationary gear signal into a finite number of stationary intrinsic mode functions. The EEMD and EMD methods are used to process the same data. Comparison results show that the adaptive local iterative filtering can get the gear rotation frequency and avoid the false fluctuation in the iterative filtering process. Then, the permutation entropy is used to calculate the first several intrinsic mode functions of decomposition results. And the method of gray correlation degree is used to identify the different gear fault types. Results show that permutation entropy can really represent the characteristics of different fault types.

\section{Data Availability}

The data used to support the findings of this study are available from the corresponding author upon request.

\section{Conflicts of Interest}

The authors declare that they have no conflicts of interest to this work. The authors declare that they do not have any 
commercial or associative interest that represents conflicts of interest in connection with the work submitted.

\section{Acknowledgments}

This research was supported by the National Natural Science Foundation of China (51769007), Yunnan Local Undergraduate Universities Basic Research Joint Special Key Project (202001BA070001-002), Yunnan Application Basis Research Youth Project (2018FD089), and Yunnan Provincial Key Laboratory Construction Program (2018ZD022).

\section{References}

[1] W. Xiyang, K. Zhigao, D. Hai, and G. Tingkai, "Autoregressive model-based gear shaft fault diagnosis," Chinese Journal of Mechanical Engineering, vol. 45, no. 4, pp. 265-272, 2009.

[2] P. Fuqiang, Y. Dejie, and W. Chunyan, "Am-fm signal extraction method by the sparse signal decomposition based on multi-scale chirplet and its application to gear fault diagnosis," Chinese Journal of Mechanical Engineering, vol. 46, no. 12, pp. 1-7, 2010.

[3] N. E. Huang, Z. Shen, S. R. Long et al., "The empirical mode decomposition and the Hilbert spectrum for nonlinear and non-stationary time series analysis," Proceedings of the Royal Society of London. Series A: Mathematical, Physical and Engineering Sciences, vol. 454, no. 1971, pp. 903-995, 1998.

[4] A. Cicone, J. Liu, and H. Zhou, "Adaptive local iterative filtering for signal decomposition and instantaneous frequency analysis," Applied and Computational Harmonic Analysis, vol. 41, no. 2, pp. 384-411, 2016.

[5] Y. Deyou, W. Bo, and C. Guowei, "Extracting oscillation modes from nonstationary signals for power system using adaptive local iterative filter," Proceedings of the CSEE, vol. 36, no. 20, pp. 5431-5439, 2016.

[6] C. Baojia, W. Xinbo, Z. Chunhua, C. Fafa, Q. Guangyin, and T. Hongliang, "Fault feature extraction of rolling bearing based on ALIF and energy operator demodulation," Journal of Nanjing University of Science and Technology, vol. 42, no. 4, pp. 445-452, 2018.

[7] L. Bing, Z. Peilin, R. Guoquan, L. Dongsheng, and M. Shuangshan, "Gear fault diagnosis using empirical mode decomposition, genetic algorithm and support vector machine," Journal of Vibration, Measurement \& Diagnosis, vol. 29, no. 4, pp. 445-448, 2009.

[8] L. Tao, M. Zhuanxia, and D. Nan, "Application of multi-scale permutation entropy to fault diagnosis of scroll compressor," Journal of Lanzhou University of Technology, vol. 44, no. 1, pp. 42-46, 2018.

[9] L. Li, J. Rong, L. Taotao, Y. Jielin, M. Xiping, and G. Zewei, "Vibration fault diagnosis of wind turbine's gearbox based on multidimensional permutation entropy and SVM," Power System and Clean Energy, vol. 33, no. 5, pp. 87-91, 2017.

[10] W. Yukui, L. Hongru, and Y. Peng, "Fault identification of hydraulic pump based on multi-scale permutation entropy," China Mechanical Engineering, vol. 26, no. 4, pp. 518-523, 2015.

[11] Z. Massimiliano, Z. Luciano, and A. R. Osvaldo, "Permutation entropy and its main biomedical and econophysics applications: a review," Entropy, vol. 14, no. 8, pp. 1553-1577, 2012.

[12] L. Lin, Y. Wang, and H. Zhou, "Iterative filtering as an alternative algorithm for empirical mode decomposition,"
Advances in Adaptive Data Analysis, vol. 1, no. 4, pp. 543-560, 2009.

[13] F. Zhipeng, C. Fulv, and Z. Mingjian, Principle and Fault Diagnosis of Complex Nonstationary Signal Analysis Method for Mechanical System, Science Press, Beijing, China, 2018.

[14] Z. Wenbin, J. Jie, Y. Libin, G. Dewei, M. Jie, and P. Yasong, "Application of complementary ensemble empirical mode decomposition and singular value energy spectrum in wind power gear fault identification," Acta Energiae Solaris Sinica, vol. 41, no. 2, pp. 137-143, 2020.

[15] Z. Wenbin, G. Dewei, P. Yasong, T. Ruijing, W. Peng, and S. Yanping, "Harmonic window decomposition sample entropy and grey relation degree in rotor fault recognition," Proceedings of the CSEE, vol. 33, no. 21, pp. 132-137, 2013. 